\title{
Social Distancing of Depressive and Panic Disorders in an International Sample of Social Work Students
}

\author{
Donna S. Wang \\ Scott Smith \\ Chris R. Locke
}

\begin{abstract}
Using an international sample, this study examined what variables were associated with social distancing among social work students. A total of 1,042 students from seven universities in the United States, United Kingdom, and Australia participated in a cross-sectional paper and pencil survey that applied the Social Distancing Scale to case vignettes describing an individual with panic disorder or major depressive disorder. The results show that levels of social distancing were related to age, knowing someone with a mental illness, type of disorder, level of conservatism, race, country, professional interest in mental health, level of student, and sex. Overall, $16.8 \%$ of the variance was accounted for with these significant variables. Implications for social work education and future research are discussed.
\end{abstract}

Keywords: Stigma, mental health, mental illness, social distance, social work education, international social work

Stigma, as with any social construct, is multifaceted and complex. The MerriamWebster's Dictionary defines it as "a mark of shame or discredit; a stain, or an identifying mark or characteristic and when associated with mental illness, involves feelings, attitudes and behaviors" (Penn \& Martin, 1998, cited in Overton \& Medina, 2008). There are several theories of stigma, such as social identity theory, which describes how people use social constructs to judge or label someone who is different or disfavored (Overton \& Medina, 2008).

From a psychological perspective, the process in which one stigmatizes involves four social-cognitive processes: cues, stereotypes, prejudice, and discrimination (Corrigan, 2004). This process of stigmatizing is described further by Overton and Medina (2008) as the following: the cue as a social cognitive process in which a person recognizes internally that something is different about an individual, which then activates a stereotype. If the stereotype is negative, it is considered a prejudice. Lastly, that prejudice can then lead to a behavioral response, often discrimination or social distancing. Within the four processes of stigmatizing, the first three are internal responses, and the fourth, a behavioral response, is external.

Although stigma and social distancing are not interchangeable or synonymous, there is a relationship between the two. Social distancing can be one behavioral consequence of stigmatizing attitudes. Because social distancing is manifest and not latent, it is often used by researchers as a measurable indicator of possible stigma (Covarrubias \& Han,

Donna S. Wang, Ph.D., MSW, is an Associate Professor in the Social Work Department at Long Island University, Brooklyn Campus; Scott Smith, PhD, LCSW, is and Assistant Professor in the School of Social Work at Texas State University in San Marcos; Chris R. Locke, Ph.D., LCSW, is a social worker in Fort Pierce, FL..

Copyright (C) 2014 Advances in Social Work Vol. 15 No. 2 (Fall 2014), 480-494 
2011; Faulkner, Irving, Paglia-Beck, \& Adlaf, 2010; Feldman \& Crandell, 2007; Link, Yang, Phelan, \& Collins, 2004; Reavley \& Jorm, 2011).

Measuring an increased desire for social distance is considered an important measure of stigma (Couture \& Penn, 2003) as social distancing can have consequences for employment for those with mental illness. For example, those with mental illness can experience employer discrimination during the hiring process (Feldman \& Crandall, 2007; Overton \& Medina, 2008). One study conducted with social work students found that the majority $(78.9 \%)$ said they would not hire a person with severe and persistent mental illness as a babysitter for their child (Covarrubias \& Han, 2011). This form of employer stigma (i.e., not hiring one for a job) is an example of social distancing or, put another way, a lack of desire to include people with serious mental illness in one's personal network (Link et al., 2004). However, it should also be noted that social distancing can also occur for reasons other than stigma, such as fear (Theriot \& Lodato, 2012).

Unfortunately, mental healthcare service providers are not excluded from stigmatizing and social distancing of clients. Provider stigma can be defined as "the negative attitudes, beliefs, and behaviors that mental health providers possess and enact toward clients they serve, even unknowingly or subtly" (Charles, 2013, p. 361). Mental healthcare providers, including social workers, may harbor prejudices against those with mental illness (Covarrubias \& Han, 2011), which may pose a threat to the effective provision of social services. Moreover, although social workers are one of the largest providers of mental health services in the United States, few studies have explored social work students' attitudes towards mental illness (Theriot \& Ladato, 2012). Theriot and Ladato (2012) stated that at the time of their contribution, only one study (Eack \& Newhill, 2008) had specifically looked at social work students and their attitudes towards mental illness. Thus, the attitudes toward mental health clients of social work students, who are future practitioners, are largely unknown. Service delivery to individuals with mental illness or any other vulnerable population is integral to the social work profession. This responsibility to our clients requires social workers to perform effectively and ethically, which requires awareness of one's own inherent biases. The purpose of this current study was to investigate what personal factors among social work students are associated with social distancing towards individuals with mental illness, and more specifically, depression and anxiety. Social distancing was chosen because it is manifest rather than latent, and it may have a direct impact on how clients are treated.

\section{Rationale}

Past research has been suggested that it would be beneficial to disaggregate various mental illnesses to determine differences of mental health stigma (Covarrubias \& Han, 2011). Much of the literature around social distancing focuses on schizophrenia (e.g., Faulkner et al., 2010; Reavley \& Jorm, 2011) and other mental illnesses perceived as dangerous. Reavley and Jorm (2011) state that it may not appropriate to generalize stigmatizing attitudes about schizophrenia to anxiety disorders, and that there is a need to explore specific attitudes towards anxiety disorders. Thyer and Wodarski (2007) also noted that panic disorder, a type of anxiety disorder, is relatively common in the general 
population. These conditions are not generally thought of as dangerous, and individuals experiencing these conditions function fairly well in the community, as opposed to conditions typically viewed as "dangerous (also known as peril)," such as schizophrenia (Corrigan, 2005). Thus, for this study, both panic and major depressive disorders were chosen for four reasons: 1) these disorders represent illnesses from the most common diagnostic categories: anxiety disorders (Sadock \& Sadock, 2003) and mood disorders (Corrigan, Mueser, Bond, Drake, \& Solomon, 2008), and thus are common conditions that social workers encounter and treat; 2) these conditions are not typically "associated" with violence, so subtle and covert social distancing may be present; 3) to compare anxiety and depression and their underlying assumptions as suggested by Reavley and Jorm (2011); and 4) to expand the literature around stigma and social distancing to include additional disorders.

Stigma may operate differently according to variables associated with social distancing and attitudes, such as age, gender, and culture (Reavley \& Jorm, 2011). Because stigma is a multi-dimensional construct, the researchers included in this study variables thought to be associated with social distancing and attitudes, based on past research and theory. Variables in the current study included demographics such as age, race, and gender (Covarrubias \& Han, 2011; Faulkner et al., 2010; Theriot \& Ladato, 2012). The study also included other personal and educational factors thought to be related to attitudes in general, such as increased contact (Covarrubias, \& Han, 2011, Cummings, Alder, \& DeCoster, 2005), student interest in a practice area (Kane, 2004), graduate or undergraduate level of a student (Curl, Simons, \& Larkin, 2005; Theriot \& Ladato, 2012; Wang, Ihara, Chonody, \& Krase, 2012), knowing someone (Cummings, Alder, \& DeCoster, 2005; Kane, 2004), religion and importance of religion (Anderson \& Wiscott, 2003), and country (Angermeyer, Holzinger, Carta, \& Schomerus, 2011). Because sexual orientation and level of conservatism are also believed to be related to attitudes and perspectives, they were also included.

The researchers of this current study also chose to include an international sample of social work students. Mental health can be conceptualized differently among various cultures and may impact career choices. For example, Limb and Organista (2003) found that students of color and American Indians were more likely than Whites to choose poverty as their area of practice. This finding may reflect a priority of social justice and advocacy over direct mental health services among various racial and cultural groups. Thus to explore various differences, our study included an international sample of social work students from three Westernized countries (US, UK, and Australia) in our study. Although diversity would be better investigated by examining differences among cultures within and between countries of varying financial and economic development, the similarities and differences that exist between groups in these Westernized countries could help to clarify cultural differences and perhaps lead to larger-scale studies that include both Eastern and Western countries.

\section{Method}

The data for this study were collected from September 2010 through December 2011 from five universities in the US, one suburban university in the UK, and one suburban 
university in Australia. All of the universities were home institutions of the research team at that time. Of the universities located in the United States, three of the universities were located in large cities (New York, NY and Philadelphia, PA) and two were located in the rural South (Texas and Alabama), and were chosen for both access as well as an effort to diversify the sample in terms of rural/urban, religion, and culture. Approvals were obtained from all the universities' institutional review boards (or its equivalent). This study was a cross-sectional, self-administered, in-person paper and pencil survey that targeted social work students. Members of the research team or research assistants recruited students from various undergraduate and graduate social work classes. Course schedules were obtained and instructors were contacted for permission to administer the survey during class time. Attempts were made to administer the survey in as many of the classes as possible. Students were instructed that if they already completed the survey in another class they were not to fill it out again. Students were given the option of completing the survey during the time it was administered or returning it to the investigator in his/her faculty mailbox at a later time. The survey took approximately 1015 minutes to complete.

For this study, stigma was conceptualized as social distancing from those with mental illness and the conditions targeted were panic disorder and major depressive disorder. Social distancing is used as a proxy for behavior to help approximate how students would respond to these individuals. The inclusion of social distance as a dependent variable was first introduced when Bogardus (1925) examined the construct in relation to race and ethnicity. Bogardus' scale was later modified to be more applicable to the mental health population (Link, Cullen, Frank, \& Wozniak, 1987). Vignettes are utilized in the present study in order to better understand how attitudes differ based on the diagnostic label. Vignettes have been used in numerous studies related to mental health stigma (Feldman \& Crandall, 2007), including the General Social Survey (Link, Phelan, Bresnahan, Stueve, \& Pescosolido, 1999; Pescosolido et al., 2010). The advantages of using vignettes as an independent variable in stigma research have been well documented and permit the researcher to provide a more thorough description of an individual as opposed to simply presenting a diagnosis (Link et al., 2004). Moreover, a diagnosis is typically more methodologically rigorous than generic references to "mental illness" as a descriptor.

Two researcher-created vignettes (one for panic disorder and one for major depressive disorder) were used that were consistent with the criteria of the Diagnostic and Statistical Manual of Mental Disorders IV-TR (DSM-IV-TR; American Psychiatric Association, 2000). The following is the vignette developed to reflect a client experiencing panic disorder:

For the past two months, Mike has been feeling very nervous almost every day. There are times during the day when he has trouble catching his breath and fears he is losing control. During these times, he also reports sweating and trembling. Mike has had to leave work early a few days because of this. He frequently worries about having future "attacks" because they seem to come about for no reason. Mike has also been having difficulty sleeping and has become more moody. 
The following is the vignette developed to reflect a client experiencing major depressive disorder:

For the past two months, Mike has been feeling very sad almost every day. He often feels fatigued and does not feel like doing things with friends that he used to enjoy. He has arrived late to work a few days because he barely had enough energy to get out of bed. Mike often has trouble sleeping at night. During the day, he feels worthless and feels guilty about things that are not really his fault. Sometimes, when Mike feels down, he will eat a lot more food than usual, which has resulted in weight gain.

\section{Measures}

Dependent variable: Social Distance Scale (SDS). Each student was randomly given either the major depressive disorder vignette or the panic disorder vignette that applied the SDS. The version used in this study was adapted from one created by Link and colleagues (1987), which had an internal consistency reliability (or Cronbach's alpha) estimated at .92. Students in the present study were asked how willing they would be to: 1) rent a room to the client, 2) have him as a coworker, 3) have him as a neighbor, 4) have him as a caretaker for the respondent's children, 5) have him marry the respondent's child, 6) have him date a friend of the respondent (the original item is stated as "introduce him to a young woman". This was re-worded in the current study in order to not bias heterosexuality), and 7) recommend that he work for a friend of the respondent. A fivepoint Likert scale was utilized with higher scores indicating greater social distancing (i.e., a less favorable attitude); lower scores indicating less social distancing (i.e., a more favorable attitude). A total composite score was calculated, creating a possible range of 7 to 35 with a higher score indicating a higher level of social distancing (Corrigan, Edwards, Green, Diwan, \& Penn, 2001). For this current study, Cronbach's alpha was estimated at $83.9 \%$.

Independent variables. The independent variables were age, asked as "What is your age?"; whether or not they know someone with a mental illness (yes/no); diagnosiswhether they received the depression or anxiety vignette; what they consider their social political beliefs to be (mostly liberal, slightly liberal, moderate, slightly conservative, and mostly conservative were collapsed and treated as nominal level variables: liberal, moderate, and conservative); country (USA, UK, or Australia); whether or not they had a primary social work interest in mental health (yes/no); level of the student (graduate or undergraduate); sex (choices given were male, female, and intersexed/transgender. Due to cell sizes, these were collapsed into male and female); sexual orientation (choices were gay/lesbian, bisexual, heterosexual, neither hetero- or homosexual, and unsure. Due to cell sizes, these were dichotomized into heterosexual and sexual minority); race (seven options were offered, but they were collapsed into four categories: White, Black, Hispanic/Latino(a), and Other); religious affiliation (several choices were offered, but they were collapsed into not religious at all, spiritual only, and having a religious affiliation). The importance of religious/spiritual beliefs was asked with choices offered of very important, important, moderately important, of little importance, and 
unimportant, again measured on a five-point Likert scale with a higher number indicating a higher level of importance.

\section{Results}

A total of 1,042 students participated in this study. The majority of the participants in the sample $(94.8 \%)$ were social work students or majors; however, all students were included because they were enrolled in a social work class and may not yet have declared their majors. Undergraduates comprised most of the sample $(63.3 \%, \mathrm{n}=653)$, with the average age reported as 27.44 years $(\mathrm{SD}=9.04)$. Respondents were mostly from the USA $(80.1 \%, \mathrm{n}=835)$ followed by the UK $(10.2 \%, \mathrm{n}=106)$ and Australia $(9.7 \%, \mathrm{n}=101)$. The sample was mostly female $(86.4 \%, \mathrm{n}=894)$; race/ethnicity was mostly White $(57.1 \%, \mathrm{n}=595)$, followed by Black $(22.5 \%, \mathrm{n}=234)$, Other $(11 \%, \mathrm{n}=110)$, and then Hispanic/Latino(a) $(5.8 \%, \mathrm{n}=58)$. The respondents tended to be more liberal, with $53.9 \%$ of the sample identifying themselves as mostly or slightly liberal. Finally, the sample was primarily heterosexual $(89.1 \%, \mathrm{n}=928)$.

Most of the respondents $(84.6 \%, \mathrm{n}=882)$ indicated that mental health was not their primary interest in practice, with $15.4 \%(\mathrm{n}=160)$ indicating that working in mental health was their intended area of practice. The majority $(68.8 \%, n=717)$ reported that they knew someone with a mental illness, and the average social distancing score for the overall sample was $22.57(\mathrm{SD}=5.28)$. Anxiety $(50.6 \%, \mathrm{n}=527)$ and depression $(49.3 \%$, $\mathrm{n}=514$ ) vignettes were approximately evenly distributed (See Table 1 for sample characteristics).

A hierarchal linear regression was conducted to test which factors were associated with social distancing. The categorical variables were dummy coded to improve interpretation: level of student $(1=$ undergraduate, $0=$ graduate $)$, whether or not they knew someone with a mental illness $(1=$ yes, $0=$ no), diagnosis $(1=$ anxiety, $0=$ depression), sexual orientation $(1=$ heterosexual, $0=$ sexual minority $)$, sex $(1=$ female, 0 $=$ male $)$, race $(1=$ White, $0=$ non-White $)$, country $(1=$ United States, $0=$ Other $)$, importance of religion $(1=$ yes, $0=$ no), and whether or not they identified mental health as their intended area of practice $(1=$ no, $0=$ yes $)$. The three levels of the political affiliation and the religious affiliation variables were separated into three separate dummy variables (e.g., liberal $=1,0=$ other).

There were sixteen variables inserted into the hierarchical regression model (Model 1) that included variables that were theoretically linked to social distancing and each subsequent model only included variables that remained significant. Table 2 represents the final three models. In the first model, the liberal and spiritual variables were removed, and the moderate, sexual orientation, religious importance, not religious or spiritual, and religious affiliation variables were not significant, and therefore removed. Importance of religious beliefs, liberal political affiliation, sexual orientation, spiritual variable, not religious or spiritual variable, and religious affiliation variables were all not significant in the model. These variables were removed leaving age, knowing someone, diagnosis, political affiliation, White, country (United States), interest in mental health, gender, and level of student (undergraduate) in Model 2 to be tested. 
Table 1. Characteristics of the Sample

\begin{tabular}{|c|c|c|c|c|}
\hline Variable & $M$ & $S D$ & $\%$ & $n^{\mathrm{a}}$ \\
\hline Age (range 18-64) & 27.44 & 9.04 & & 1,023 \\
\hline \multicolumn{5}{|l|}{ Sex } \\
\hline Male & & & 13.1 & 135 \\
\hline Female & & & 86.8 & 894 \\
\hline \multicolumn{5}{|l|}{ Race } \\
\hline African American/Black & & & 23.5 & 234 \\
\hline Hispanic & & & 5.8 & 58 \\
\hline Caucasian/White & & & 59.7 & 595 \\
\hline Other & & & 11.0 & 110 \\
\hline \multicolumn{5}{|l|}{ Political Affiliation } \\
\hline Liberal & & & 53.9 & 545 \\
\hline Moderate & & & 33.2 & 336 \\
\hline Conservative & & & 12.9 & 130 \\
\hline \multicolumn{5}{|l|}{ Diagnosis (Anxiety/Depression) } \\
\hline Anxiety & & & 50.6 & 527 \\
\hline \multicolumn{5}{|l|}{ Know someone with MH issues } \\
\hline Yes & & & 68.8 & 717 \\
\hline \multicolumn{5}{|l|}{ Mental Health Interest (yes/no) } \\
\hline No & & & 84.6 & 882 \\
\hline \multicolumn{5}{|l|}{ Country } \\
\hline Australia & & & 9.7 & 101 \\
\hline United States & & & 80.1 & 835 \\
\hline United Kingdom & & & 10.2 & 106 \\
\hline \multicolumn{5}{|l|}{ Religious Affiliation } \\
\hline Not religious or spiritual & & & 13.1 & 133 \\
\hline Spiritual only & & & 23.6 & 239 \\
\hline Religious affiliation & & & 63.3 & 641 \\
\hline \multicolumn{5}{|l|}{ Sexual Orientation } \\
\hline Heterosexual & & & 89.1 & 928 \\
\hline Sexual Minority & & & 10.9 & 114 \\
\hline \multicolumn{5}{|l|}{ Undergraduate/Graduate } \\
\hline Undergrad & & & 63.3 & 653 \\
\hline STIGMA Total & 22.57 & 5.28 & & 957 \\
\hline
\end{tabular}


Table 2. Summary of Regression for the Social Distancing Scale

\begin{tabular}{|c|c|c|c|c|c|c|c|c|c|}
\hline \multirow[b]{2}{*}{ Variables } & \multicolumn{3}{|c|}{ Model 1} & \multicolumn{3}{|c|}{ Model 2} & \multicolumn{3}{|c|}{ Model 3} \\
\hline & $B$ & $S E B$ & $\beta$ & $B$ & $S E B$ & $\beta$ & $B$ & $S E B$ & $\beta$ \\
\hline Age & .054 & .021 & $.094 *$ & .051 & .021 & $.088 *$ & .050 & .021 & $.087^{*}$ \\
\hline Know someone & -1.933 & .380 & $-.171 * * *$ & -1.925 & .377 & $-.170 * * *$ & -1.965 & .376 & $-.174 * * *$ \\
\hline Diagnosis: depression & 2.215 & .331 & $.218^{* * *}$ & 2.296 & .331 & $.225^{* * *}$ & 2.302 & .331 & $.225 * * *$ \\
\hline \multicolumn{10}{|l|}{ Liberal $^{\mathrm{a}}$} \\
\hline Moderate & .517 & .382 & .047 & -.478 & .373 & .201 & & & \\
\hline Conservative & 1.147 & .528 & $.077^{*}$ & 1.185 & .512 & $.079 *$ & 1.011 & .493 & $.067 *$ \\
\hline White & -.959 & .396 & $-.087 *$ & -.945 & .386 & $-.086^{*}$ & -.985 & .385 & $-.089 *$ \\
\hline United States & 2.517 & .507 & $.178 * * *$ & 2.250 & .487 & $.159 * * *$ & 2.235 & .487 & $.158 * * *$ \\
\hline Non-MH Interest & .772 & .483 & $.054 *$ & .995 & .478 & $.070^{*}$ & 1.018 & .477 & $.071 *$ \\
\hline Undergraduate & -.897 & .395 & $-.084 *$ & -.809 & .393 & $-.076^{*}$ & -.784 & .393 & $-.073^{*}$ \\
\hline Female & 1.183 & .510 & $.076^{*}$ & 1.017 & .507 & $.065^{*}$ & 1.039 & .507 & $.067 *$ \\
\hline Sexual Orientation & .101 & .557 & .006 & & & & & & \\
\hline Religious importance & -.014 & .153 & -.004 & & & & & & \\
\hline \multicolumn{10}{|l|}{ Spiritual $^{\mathrm{a}}$} \\
\hline Not religious or spiritual & .936 & .700 & .056 & & & & & & \\
\hline Affiliation & .336 & .411 & .031 & & & & & & \\
\hline$R^{2}$ & \multicolumn{3}{|c|}{.415} & \multicolumn{3}{|c|}{.406} & & \multicolumn{2}{|c|}{.404} \\
\hline F change in $R^{2}$ & \multicolumn{3}{|c|}{$11.798 * * *$} & \multicolumn{3}{|c|}{$15.935^{* * *}$} & & \multicolumn{2}{|c|}{$17.51 * * *$} \\
\hline
\end{tabular}

Notes: ${ }^{\text {a }}$ Removed from model; ${ }^{*} p<.05,{ }^{* *} p<.01, * * * p<.001$ 
In the second model, only the political affiliation of moderate was not significant so it was removed from the model. Model 3 included the following significant variables: age, know, diagnosis, conservative, race, country, mental health interest, level of student, and sex. When tested, all variables were significant and no items were flagged for removal indicating that the final model was Model 3, accounting for $16.3 \%$ of the variance.

\section{Discussion}

Overall, the participants reported more social distancing towards major depressive disorder than towards panic disorder. It is not entirely clear why student participants viewed anxiety more favorably than depression; therefore the interpretations are offered with caution. However, one possible explanation could be that anxiety and panic are more socially accepted than depression. A second possible explanation is that anxiety and panic are more consciously experienced than depression, in that people often recognize when they are anxious about day-to-day events. Thus, this could result in a normalization of these emotions. A third explanation is that a depressive disorder may be perceived as more severe than a panic disorder.

Another potential explanation for the difference in attitudes between depression and panic disorder is the link between depression and excessive reassurance seeking (ERS) behavior and resulting interpersonal rejection (Evraire \& Dozois, 2011; Starr \& Davila, 2008). In other words, individuals with depression may constantly seek reassurance from friends and family that that they are loved and cared about, which can unknowingly lead to "burning out" their support system. Thus, it is possible that participants in the present study endorsed more favorable attitudes (i.e., less interpersonal rejection) toward the panic disorder vignette because they do not associate people with anxiety as constantly seeking reassurance as a person with depression might.

The difference between depression and anxiety do suggest that further inquiry into differentiation of various conditions is critical in understanding how to address stigma and social distancing. For example, when studying severe and persistent mental illness, Covarrubias and Han (2011) found that depression was less stigmatized than bipolar disorder or schizophrenia, suggesting that depression may be more socially acceptable than the others. This comparison has been substantiated elsewhere (Mann \& Himelein, 2004, cited in Theriot \& Ladato, 2012). These disparities clearly exist between various conditions and have direct implications for social work practice, values, and ethics. Deeper inquiry into these disparities will help to determine strategies and interventions at both the educational and workforce levels. The various causes of social distancing would determine approaches to amelioration. For example, educational efforts would differ if depression is seen as more stigmatizing based on fear of dangerousness rather than a result of burnout for excessive reassurance. Additionally, continual introspection into managing one's own personal biases may need to be encouraged and taught in order to not reduce detrimental effects to individuals with mental illness.

Comparisons have been made in the past between Western and non-Western countries, and it was found that certain cultures held less favorable attitudes towards mental illness, possibly based on notions of guilt and individual blame (Angermeyer et 
al., 2011). Our results found that the students from United States exhibited greater social distancing than the students from the UK and Australia. Given that all the countries in this current study were Westernized, it is interesting that these differences were found. One factor may be that the US is less homogenized and more diversified than the UK or Australia. Additionally, cultural or policy influences may contribute to the perception or treatment of people with mental illness. As provision of mental health services may vary from country to country, social workers in other countries may play less of a role in direct assessment and treatment than they do in the United States. For example, in the UK services for less severe conditions are generally provided by a general medical practitioner.

Our study found differences between sexes, which is in congruence to previous the finding that women exhibited greater social distancing than males (Chung, Chen, \& Liu, 2001, cited in Theriot \& Ladato, 2012), and contrary to finding that women were more tolerant and sympathetic to mental illness (Hinkelman \& Granello, 2003, cited in Theriot \& Ladato, 2012), and no differences between sexes (Covarrubias \& Han, 2011). The contradiction in previous findings warrants closer attention to determine under which circumstances sex contributes to social distancing and stigma. One possible explanation for our findings is that the majority of our sample were female $(86.8 \%)$, whereas the vignette presented featured "Mike," a male. A gender bias towards males with mental health issues may account for some of the findings. Another possibility might be that male social work students are not representative of males in the general population (e.g., they might be more accepting of persons with SMI). Future research can seek to include either gender neutral vignettes, vignettes with both sexes, or vignettes with randomized sexes to make further distinctions. Additionally, to the authors' knowledge, this current study was one of the first to include sexual orientation, which was also found to be unrelated to levels of social distancing. The logic that sexual minorities are often stigmatized themselves and thus are less stigmatizing of mental illness was not supported in our findings.

Past research has suggested that examining a practitioner's past professional and/or personal experiences with mental health services would be useful (Charles, 2013). Knowing someone with a mental illness as well as having an interest in mental health were both related to lower levels of stigma in our current study. These findings support that perspectives are not "fixed" (e.g., demographic variables such as age or gender) and solutions can be offered to correct stigmatizing attitudes. It may not be surprising that students interested in pursuing a career in the mental health field viewed mental health clients as more socially acceptable than those who do not desire a career path in mental health. In other words, in general, those students who possessed negative attitudes toward mental health clients would most likely not express an interest in wanting to work directly with such clients. Exposure has been cited as a mechanism to increase interest, as in the study that showed that individuals who have more knowledge about and contact with people with schizophrenia had more positive attitudes (Eack \& Newhill, 2008) and in another study that demonstrated the effectiveness of the use of contact to reduce stigma in the construction of anti-stigma interventions (Corrigan, 2005). These findings show 
promise and opportunities for the profession to implement ways of reducing stigmatizing attitudes.

Graduate level students showed more social distancing than undergraduate students. A similar finding was that new social work students generally held more positive attitudes towards serious mental illness than did other students (Theriot \& Ladato, 2012). Although undergraduates have patterns of concerns that differ from those in the general population (Feldman \& Crandall, 2007), the findings that undergraduate students and those who know someone with a mental illness displayed less social distancing support the idea that early exposure to intervention and education about mental illness could help to reduce negative stereotypes about mental illness (Theriot \& Ladato, 2012). Additionally, understanding the trajectory of career choices among students may shed light to how ideas and interests are formed.

The findings of our study have other implications for practice and social work education. Because the factors related to social distancing were largely related to nonfixed demographics such as being an undergraduate student, knowing someone with mental illness, and having an interest in mental health, anti-stigma education efforts may be helpful in correcting stigmatizing attitudes (Covarrubias \& Han, 2011). For example, exposure to individuals with mental illness who exhibit a range of functioning may demonstrate that mental illness is not always debilitating. This may serve as a model for students' subsequent contact with clients in the field. Further, efforts earlier rather than later in social work education could help students formulate interest and understanding in working with individuals with mental illness. Perhaps exposure even prior to field placement may be helpful.

Second, various attitudes towards different mental health conditions and different levels of severity of mental illness may also be useful in determining where and why certain biases exist. It has been noted that social workers may be disillusioned because of exposure to clients who are experiencing the most severe symptoms of mental illness during periods of decomposition (Covarrubias \& Han, 2011). This idea may be comparable to death anxiety among geriatric social workers, as Greene (1984) found that geriatric social workers had higher levels of death anxiety than non-geriatric social workers and greater number of years in the field was correlated with a higher level of death anxiety (although this leveled off after six or seven years). This supports the notion that working with individuals with mental illness may result in seeing severe and persistent behaviors as norms. This phenomenon is also called the "clinician's illusion", which is the belief that people with severe mental illness are always low functioning; cannot get along in society; and exhibit socially undesirable behaviors, like being violent (Cohen \& Cohen, 1984, as cited by Covarrubias \& Han, 2011). Social workers may displace their own biases onto their clients causing harm that could be curbed through further education or training. Addressing issues of counter-transference could also help to reduce stigmatizing attitudes (Ahmedani, 2011). This may be critical prior to exposure in the field to clients with severe mental illness. Social work pedagogy that addresses these deficiencies in exposure, diversity, and training should be evaluated. 
There are some limitations to our study that need to be highlighted. One limitation is that we were only able to simply describe social distancing given the cross-sectional nature of our study. We were not able to explain how or where perceptions of mental illness were formed, the impact of stigma in terms of a person's behavior, or the treatment of clients with mental illness. How stigma and social distancing actually affect direct practice is an important research issue. A second limitation of our study is that although this is one of the few studies that includes a diverse sample in terms of urban/rural and different countries, the way the survey was administered still limits generalizability: we cannot be certain that a student did not complete the survey twice, or that students did not report in a way that was socially desirable. Lastly, another limitation of our study is that it only compared major depressive disorder and panic disorder. Other forms of severe and persistent mental illnesses were not assessed or compared. Further research that explores the relationships between stigma or social distancing and various mental illnesses is warranted. Replicating the current study with students or the general public would help to advance the stigma literature by potentially supporting this current study's findings or identifying other explanations for the differences in attitudes based on disorder type.

In sum, the findings that undergraduate students, those with an interest in mental health, and those who knew someone with a mental illness all showed less social distancing indicate a potential for opportunities to create meaningful experiences within social work practice and education. These factors that are associated with social distancing all suggest ways we can intervene to increase awareness and sensitivity towards individuals with mental illness. Social workers play an extremely important role in treating and advocating for consumers with mental illness. One study found that social workers held the second lowest amount of stigma (behind psychiatric nurses/nursing students) among seven professional groups (Kassam, Papish, Modfill, \& Patten, 2012). We further our mission of improving the lives of individuals who are vulnerable if we are able to ensure that social workers possess destigmatizing attitudes towards clients and can educate and advocate within the general population and policy arenas.

\section{References}

Ahmedani, B. (2011). Mental health stigma: Society, individuals and the profession. Journal of Social Work Values \& Ethics, 8(2), 4-1-4-16.

American Psychiatric Association [APA]. (2000). Diagnostic and statistical manual of mental disorders $\left(4^{\text {th }}\right.$ ed., Text rev.). Washington, DC: Author.

Anderson, D., \& Wiscott, R. (2003). Comparing social work and non-social work students' attitudes about aging: Implications to promote work with elders. Journal of Gerontological Social Work, 42(2), 21-36.

Angermeyer, M., Holzinger, A., Carta, M., \& Schomerus, G. (2011). Biogenetic explanation and public acceptance of mental illness: Systematic review of population studies. British Journal of Psychiatry, 199, 367-372. 
Bogardus, E. S. (1925). Social distance and its origins. Journal of Applied Sociology, 9, 216-226.

Charles, J. (2013). Mental health provider-based stigma: Understanding the experience of clients and families. Social Work in Mental Health, 11(4), 360-375.

Corrigan, P. (2004). How stigma interferes with mental health care. American Psychologist, 59(7), 614-625.

Corrigan, P. W. (Ed.). (2005). On the stigma of mental illness. Washington, DC: American Psychological Association.

Corrigan, P. W., Edwards, A. B., Green, A., Diwan, S. L., \& Penn, D. L. (2001). Prejudice, social distance, and familiarity with mental illness. Schizophrenia Bulletin, 27(2), 219-225.

Corrigan, P. W., Mueser, K. T., Bond, G. R., Drake, R. E., \& Solomon, P. (2008). Principles and practice of psychiatric rehabilitation: An empirical approach. New York, NY: Guilford Press.

Couture, S. M., \& Penn, D. L. (2003). Interpersonal contact and the stigma of mental illness: A review of the literature. Journal of Mental Health, 12, 291-305.

Covarrubias, I., \& Han, M. (2011). Mental health stigma about serious mental illness among MSW students: Social contact and attitude. Social Work, 56(4), 317-325.

Cummings, S. M., Alder, G., \& DeCoster, V. A. (2005). Factors influencing graduate social work students' interest in working with elders. Educational Gerontology, 31, 643-655.

Curl, A. L., Simons, K., \& Larkin, H. (2005). Special section: Innovations in gerontological social work education factors affecting willingness of social work students to accept jobs in aging. Journal of Social Work Education, 41, 393-406.

Eack, S. M., \& Newhill, C. E. (2008). An investigation of the relations between student knowledge, personal contact, and attitudes toward individuals with schizophrenia. Journal of Social Work Education, 44, 77-95.

Evraire, L. E., \& Dozois, D. J. (2011). An integrative model of excessive reassurance seeking and negative feedback seeking in the development and maintenance of depression. Clinical Psychology Review, 31(8), 1291-1303.

Faulkner, G., Irving, H., Paglia-Beck, A., \& Adlaf, E. (2010). Adolescent knowledge of schizophrenia and social distancing: A province-wide survey. Journal of Community Psychology, 38(8), 933-942.

Feldman, D., \& Crandall, C. (2007). Dimensions of mental illness stigma: What about mental illness causes social rejection? Journal of Social \& Clinical Psychology, 26(2), 137-154.

Greene, R. (1984). Ageism, death anxiety and the caseworker. Journal of Social Service Research, 7, 55-69. 
Kane, M. (2004). Predictors for future work with elders. Journal of Gerontological Social Work, 42(3/4), 19-38.

Kassam, A., Papish, A., Modfill, G., \& Patten, S. (2012). The development and psychometric properties of a new scale to measure mental illness related stigma by health care providers: The opening minds scale for Health Care Providers (OMSHC). BMC Psychiatry, 12(62), 1-12. doi:10.1186/1471-244X-12-62.

Limb, G., \& Organista, K. (2003). Comparisons between Caucasian students, students of color, and American Indian students on their views on social work's traditional mission, career motivations, and practice preferences. Journal of Social Work Education, 39(1), 91-109.

Link, B. G., Cullen, F. T., Frank, J., \& Wozniak, J. F. (1987). The social rejection of former mental patients: Understanding why labels matter. American Journal of Sociology, 92(6), 1461-1500.

Link, B. G., Phelan, J. C., Bresnahan, M., Stueve, A., \& Pescosolido, B. A. (1999). Public conceptions of mental illness: Labels, causes, dangerousness, and social distance. American Journal of Public Health, 89(9), 1328-1333.

Link, B. G., Yang, L. H., Phelan, J. C., \& Collins, P. Y. (2004). Measuring mental illness stigma. Schizophrenia Bulletin, 30(3), 511-541.

Overton, S., \& Medina, S. (2008). The stigma of mental illness. Journal of Counseling and Development, 86, 143-151.

Pescosolido, B. A., Martin, J. K., Long, J. S., Medina, T. R., Phelan, J. C., \& Link, B. G. (2010). "A disease like any other?" A decade of change in public reactions to schizophrenia, depression, and alcohol dependence. American Journal of Psychiatry, 167(11), 1321-1330.

Reavley N., \& Jorm, A. (2011). Stigmatizing attitudes towards people with mental disorders: Findings from an Australian National Survey of Mental Health Literacy and Stigma. Australian and New Zealand Journal of Psychiatry, 45, 1086-1093.

Sadock, B. J., \& Sadock, V. A. (2003). Synopsis of psychiatry ( $\left(^{\text {th }}\right.$ ed.). Philadelphia, PA: Lippincott, Williams, \& Wilkins.

Starr, D. L., \& Davila, J. (2008). Excessive reassurance seeking, depression, and interpersonal rejection: A meta-analytic review. Journal of Abnormal Psychology, 117(4), 762-775.

Theriot, M., \& Ladato, G. (2012). Attitudes about mental illness and professional danger among new social work students. Journal of Social Work Education, 48(3), 403-423.

Thyer, B. A., \& Wodarski, J. S. (Eds.). (2007). Social work in mental health: An evidence-based approach. Hoboken, NJ: John Wiley \& Sons, Inc.

Wang, D., Ihara, E., Chonody, J., \& Krase, K. (2012). Social work faculty interest in aging: Impact of education, knowledge, comfort, and experience. Gerontology and Geriatrics Education, 34, 257-271. 


\section{Author note}

Address correspondence to: Donna Wang, Ph.D., LMSW, Associate Professor, Social Work Department, Long Island University, Brooklyn Campus, 1 University Plaza, Health Sciences Building 248, Brooklyn, NY 11201. Email: donna.wang@liu.edu 